\title{
Muerte encefálica y donación de órganos en unidades de cuidados intensivos pediátricos de Argentina. Estudio multicéntrico
}

a. Servicio de Terapia Intensiva del Sanatorio Allende, Córdoba, Argentina.

b. Servicio de Terapia Intensiva del Hospital de Niños de la Santísima Trinidad (HNST), Córdoba, Argentina.

c. Servicio de Terapia Intensiva del Hospital General de Niños Dr. Pedro de Elizalde (HGNPE), Ciudad Autónoma de Buenos Aires, Argentina.

d. Servicio de Terapia Intensiva del Sanatorio Sagrado Corazón, Ciudad Autónoma de Buenos Aires, Argentina.

e Servicio de Terapia Intensiva del Hospital Infantil Municipal, Córdoba, Argentina.

f. Servicio de Terapia Intensiva del Hospital de Pediatría SAMIC "Prof. Dr. Juan P. Garrahan", Ciudad Autónoma de Buenos Aires, Argentina.

g. Servicio de Terapia Intensiva del Hospital Dr. Guillermo Rawson, San Juan, Argentina.

h. Ente Coordinador de Ablación e Implantes de Córdoba, Córdoba, Argentina.

Correspondencia: Dr. Pedro Taffarel: pedrotaffarel@hotmail. com

Financiamiento: Ninguno.

Conflicto de intereses: Ninguno que declarar.

Recibido: 24-2-2017 Aceptado: 11-7-2017

\section{Brain death and organ donation in Argentine pediatric intensive care units. A multicenter study}

\author{
Dr. Germán Bonetto, ${ }^{a, b}$ Dr. Pedro Taffarel,, c,d Dra. Melisa Gamerman, ${ }^{b, e}$ \\ Dr. Facundo Jorro Barón, ${ }^{c}$ Dra. Carolina Gaviña ${ }^{f}$ Dra. Laura Flores, ${ }^{f}$ \\ Dr. Emanuel Fernández,g Dra. Andrea Zifferman, ${ }^{d, f}$ Dr. Gustavo Debaisi, ${ }^{c}$ \\ Dr. Marcelo Acerenza, ${ }^{d, f}$ Dr. Jorge Selandari, ${ }^{f}$ Dr. Luis Landry, $f$ Dr. Ariel Cacciamano, ${ }^{e}$ \\ Dr. Alfredo Clavel, ${ }^{g}$ Dra. Patricia Capocasa, ${ }^{b}$ Dr. Bernardo Calvo ${ }^{a}$ y Dr. Marcial Angos. ${ }^{h}$
}

\section{RESUMEN}

La muerte encefálica (ME) es una condición determinada por el cese completo e irreversible de las funciones cerebrales. El mantenimiento de estas funciones vitales crea una oportunidad para la donación de órganos.

Se realizó un estudio retrospectivo en 7 unidades de terapia intensiva pediátrica de Argentina $(01 / 01 / 2013$ al 30/09/2016), para determinar la incidencia de ME clínicas y certificadas, y la proporción de trasplantes efectivos.

El 19,14\% de los pacientes fallecidos (147/768) cumplían con los requisitos clínicos de ME, siendo su principal causa el politraumatismo. En el 13,4\% de los óbitos la ME fue certificada (103), el electroencefalograma y test de apnea fueron los métodos auxiliares más utilizados. El tiempo de sostén de órganos fue de $24 \mathrm{~h}$.

Se abordaron 87 familias para el proceso de donación; se rechazaron 59 (no aptos o negativa familiar). Los donantes efectivos representaron el $25 \%$ (26/103) de los pacientes con ME certificada y 72 pacientes recibieron órganos sólidos.

Palabras clave: muerte encefálica, donación, trasplante, unidades de cuidado intensivo pediátrico.

http: / / dx.doi.org/10.5546/ aap.2018.e54

Texto completo en inglés:

http: / / dx.doi.org/10.5546/ aap.2018.eng.e54

Cómo citar: Bonetto G, Taffarel P, Gamerman M, et al. Muerte encefálica y donación de órganos en unidades de cuidados intensivos pediátricos de Argentina. Estudio multicéntrico. Arch Argent Pediatr 2018;116(1):e54-e60.

\section{INTRODUCCIÓN}

Las unidades de terapia intensiva pediátricas (UTIP) son los principales lugares donde se asiste al fallecimiento de pacientes en edad pediátrica, un porcentaje de los cuales ocurre debido a muerte encefálica (ME).

La ME es una condición determinada por el cese completo e irreversible de las funciones de los hemisferios cerebrales y del tronco encefálico, ${ }^{1}$ que es seguida, invariablemente, de paro cardíaco. Durante este proceso, sobrevienen cambios fisiopatológicos en los sistemas cardiocirculatorio y respiratorio, al igual que alteraciones metabólicas y hormonales.

El diagnóstico precoz de ME mediante el seguimiento de directrices ${ }^{2}$ (véase la Figura 1), el mantenimiento de las funciones vitales y la corrección de las alteraciones pueden crear una oportunidad para la donación de órganos.

En nuestro país, la Ley N. ${ }^{\circ} 24193$, en sus art. 23 y 24 , establece las bases para el diagnóstico de muerte bajo criterios neurológicos y le otorga al Instituto Nacional Central Único Coordinador de Ablación e Implante (INCUCAI) el carácter de organismo asesor del Ministerio de Salud y Acción Social de la Nación sobre el particular. Dicho instituto elabora un protocolo de diagnóstico de muerte bajo criterios neurológicos. ${ }^{2}$

La incidencia de ME en las UTIP es de alrededor del $16 \%$ de las muertes., La causa más frecuente son los traumatismos. $^{5}$

En el ámbito pediátrico, existe, a nivel mundial, un incremento en la necesidad de donación de órganos sólidos. Este grupo etario representa el $2 \%$ de las listas de espera en el Reino Unido y el 1,5\% en Estados Unidos de América (EE. UU.). ${ }^{6}$ La medicina 


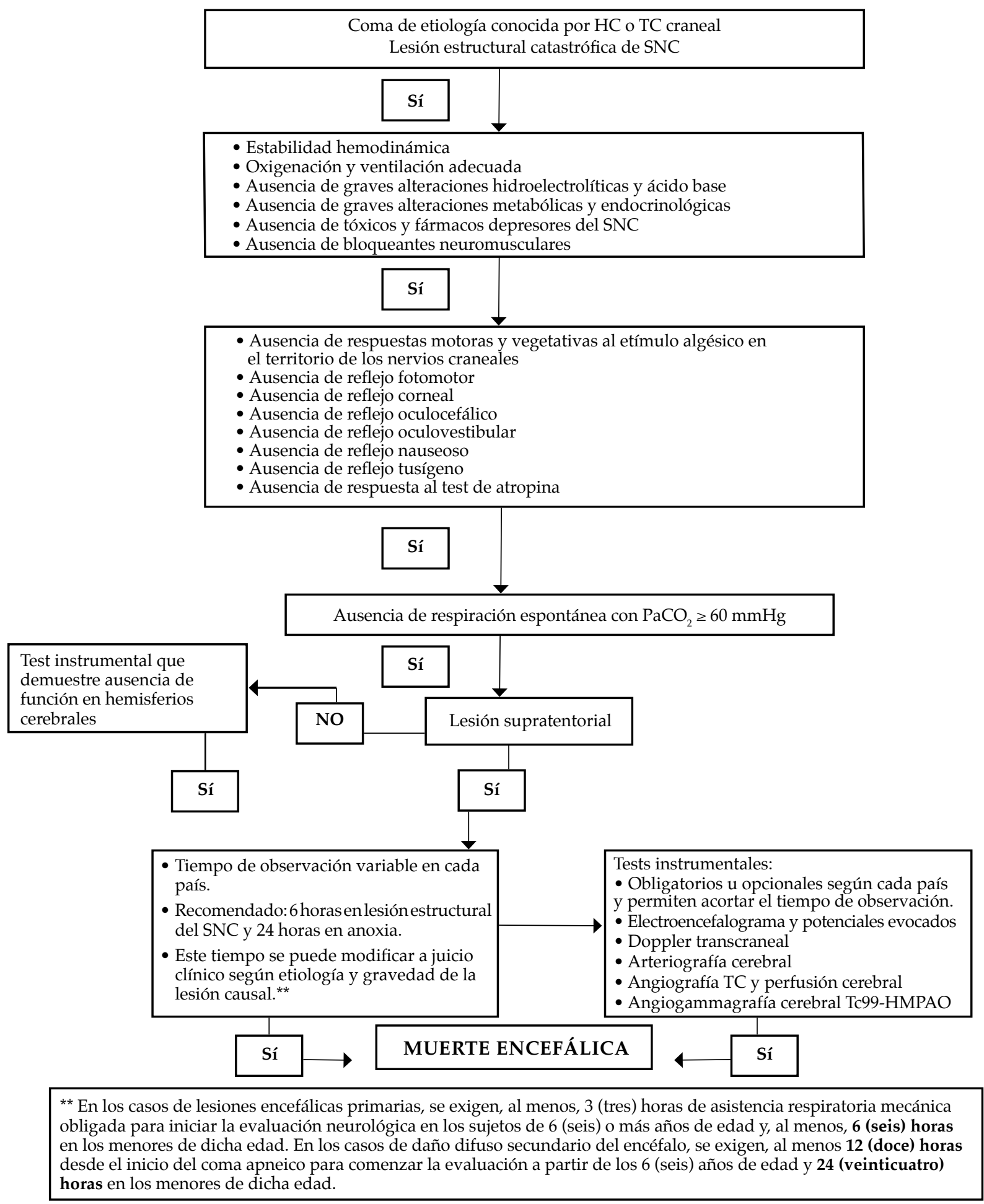

$\mathrm{HC}$ : historia clínica; TC: tomografía computada; $\mathrm{SNC}$ : sistema nervioso central; $\mathrm{PaCO}_{2}$ : presión arterial de dióxido de carbono; EEG: electroencefalograma.

Extraído y modificado de Abaroa. ${ }^{1}$ 
preventiva, la cronificación de enfermedades, el avance en el soporte vital y la escasa casuística de donantes vivos relacionados en pediatría llevan a una creciente necesidad insatisfecha de trasplantes de órganos sólidos, ${ }^{7}$ aparejada con falencias en el proceso de gestión de donación en este grupo etario. $^{8}$

Ante este panorama, la presunción precoz de ME es obligatoria a fin de activar los resortes tendientes a su certificación, y existe discrepancia en la forma de arribar a ella. ${ }^{9,10}$

En este sentido, distintas líneas de investigación se esfuerzan para optimizar el diagnóstico de muerte cerebral y el proceso de gestión de donación de órganos en su totalidad.

No existen reportes en la literatura local sobre la casuística de ME en las UTIP de Argentina ni sobre la efectividad del proceso de trasplante.

\section{OBJETIVOS}

Determinar la incidencia de ME clínicas y certificadas en UTIP.

Determinar la proporción de trasplantes efectivos sobre el total de potenciales donantes.

\section{Diseño del estudio}

Estudio retrospectivo y multicéntrico, en el período de enero de 2013 a setiembre de 2016, en las UTIP, seleccionadas por conveniencia, de los Hospitales de Niños Santísima Trinidad de Córdoba (HNST), Infantil Municipal de Córdoba (HIM), General de Niños Dr. Pedro de Elizalde (HGNPE), Nacional "Prof. Dr. Juan P. Garrahan" (HNJPG), Hospital Dr. Guillermo Rawson de San Juan y las instituciones Sanatorio Allende de Córdoba (SA) y Sanatorio Sagrado Corazón de Buenos Aires (SC).

\section{Universo y muestra}

Todos los pacientes fallecidos en las 7 UTIP en dicho período que cumplían con criterios clínicos y/o instrumentales de ME, según se muestra en la Figura 1.

\section{Metodología}

Las variables analizadas al momento del ingreso a la UTIP fueron edad (meses), sexo y motivo de ingreso.

La causa principal relacionada con la ME se agrupó en las siguientes categorías: politraumatismo (accidente en la vía pública, caída de altura, herida por arma de fuego -en esta categoría, se incluía el traumatismo craneoencefálico grave-), infección del sistema nervioso central (SNC) con germen documentado, hipoxia/isquemia cerebral cuando el mecanismo que ocasionó la ME fue la asfixia (ahogamiento, ahorcamiento) o bien como consecuencia de situaciones que llevaron a la deprivación de oxígeno $\left(\mathrm{O}_{2}\right)$ (status convulsivo, pos paro cardiorrespiratorio, muerte súbita, intoxicación por monóxido de carbono, etc.) y se observó, en una neuroimagen, lesión compatible con el mecanismo (infarto, edema generalizado, pérdida de diferenciación entre sustancias gris y blanca, etc.), accidente cerebrovascular, tumor del SNC (observados por neuroimagen), insuficiencia hepática (rango internacional normalizado -RIN- $>1,8$ o factor $\mathrm{V}<50 \%$ ) y, finalmente, la categoría "otras". También se documentó en qué proporción los pacientes con ME presentaron hipertensión endocraneana (HTE) constatada por catéter de presión intracraneana.

En los pacientes que presentaban ME certificada, se registró y analizó el tiempo transcurrido desde el ingreso a la UTIP y la certificación de ME (expresado en días), el tiempo desde la ME hasta la ablación (horas) y la estadía en la UTIP.

Los métodos auxiliares (MA) contemplados para la certificación de ME, realizados estos por el ente regulador o por la institución que albergaba al paciente, fueron test de apnea (ausencia de respiración espontánea con presión parcial de dióxido de carbono $-\mathrm{PaCO}_{2}->60 \mathrm{mmHg}$ ), electroencefalograma, potenciales evocados multimodales (somatosensitivos, auditivos del tronco cerebral y visuales con electrorretinograma) y métodos que evaluaban el flujo sanguíneo cerebral (ecodoppler transcraneal, angiografía cerebral, angiografía por tomografía computada (TC) y perfusión o angiogammagrafía cerebral Tc99-HMPAO).

Se analizaron las alteraciones inherentes a la $\mathrm{ME}$, como hiperglucemia (glucemia $>180 \mathrm{mg} / \mathrm{dl}$ ), hipotermia $\left(<35^{\circ} \mathrm{C}\right)$, diabetes insípida (poliuria, densidad urinaria $<1005$ e hipernatremia $[\mathrm{Na}>145$ meq/dl]), coagulopatía (tiempo de protrombina < $60 \%$ ) y disfunción hemodinámica (tensión arterial media $<2$ desvíos estándar-DE- de percentilo 50 para edad y/o requerimiento o aumento de las dosis de inotrópicos o vasopresores).

Finalmente, se cotejaron y analizaron los datos de los pacientes con ME con los registros del INCUCAI a fin de observar el veredicto vertido por el ente en relación con los potenciales donantes y evaluar en qué proporción se logró la donación efectiva. 
Los pacientes con ME certificada fueron catalogados como NO ABORDADOS, cuando el paro cardíaco ocurrió antes de la evaluación por parte de la entidad reguladora o bien se produjo en el proceso de evaluación, sin que dicha entidad pudiere verter un veredicto; como ABORDADOS, cuando el INCUCAI inició el proceso de evaluación considerándolos potenciales donantes y expidió un veredicto. Los pacientes abordados que fueron rechazados se agruparon como NO APTOS, si como consecuencia de su enfermedad de base o bien por la condiciones clínicas que sobrevinieron a la ME se los excluía como potenciales donantes, o NEGATIVA FAMILIAR, cuando las familias así se manifestaban.

\section{Plan de análisis}

El análisis estadístico se llevó a cabo utilizando el programa STATA 9.0 para Windows ${ }^{\circledR}$ (StataCorp, CollegeStation, Texas, USA). De acuerdo con la distribución de las variables, normal o no normal, sus valores fueron expresados en medias y DE o medianas y rango intercuartilo.

\section{RESULTADOS}

En las UTIP donde se llevó a cabo el estudio, se registraron, en el período de estudio, un total de 10541 ingresos, con una mortalidad global del 7,45\% ( $\mathrm{N}=768)$. El 19,14\% ( $\mathrm{N}=147)$ de los pacientes cumplían con los criterios clínicos de ME, y esta se certificó por MA, en 103 pacientes, correspondiente al 13,4\% del total de óbitos (véase la Figura 2). En el análisis de los datos demográficos, fueron considerados todos los pacientes con criterios clínicos de $\mathrm{ME}(\mathrm{N}=147)$; la edad mediana fue de 84 (26-144) meses.

En relación con el diagnóstico como causa de ME, la causa más frecuente fue el politraumatismo, en el 26,5\% ( $\mathrm{N}=39)$ de los casos (véase la Tabla 1).

En los pacientes con ME certificada, los días transcurridos desde el ingreso a la UTIP hasta el diagnóstico de ME fueron 2 (0,8-5); las horas de sostén de órgano fueron 24 (12-24); mientras que la estancia en la UTIP fue de $3(1,5-6)$ días.

Respecto a los MA utilizados para la certificación de ME, la combinación de electroencefalograma y test de apnea, tendiente a evaluar la corteza y el tronco cerebral, respectivamente, fue la más utilizada, ya que se empleó en el $65 \%$ de los casos $(\mathrm{N}=67)$ (véase la Tabla 1).

En cuanto a las alteraciones inherentes de la ME, estuvieron presentes en 92 de los 103 pacientes (véase la Tabla 1).; 38 (36,9\%)

TABla 1. Principales características relacionadas con el diagnóstico de muerte encefálica

\begin{tabular}{|c|c|}
\hline Variables & Números y porcentajes $(\%)$ \\
\hline \multicolumn{2}{|c|}{ Casusas de muerte encefálica en todos los pacientes con criterios clínicos $(\mathrm{N}=147)$} \\
\hline Politraumatismo & $39(26,5 \%)$ \\
\hline Hipoxia/isquemia cerebral & $37(25,2 \%)$ \\
\hline Accidente cerebrovascular & $25(17 \%)$ \\
\hline Infección del sistema nervioso central & $21(14,3 \%)$ \\
\hline Tumor del sistema nervioso central & $13(8,8 \%)$ \\
\hline Insuficiencia hepática & $7(4,7 \%)$ \\
\hline Otras & $5(3,4 \%)$ \\
\hline Pacientes con hipertensión endocraneana & $32(21,8 \%)$ \\
\hline \multicolumn{2}{|c|}{ Métodos auxiliares en los pacientes con muerte cerebral certificada $(N=103)$} \\
\hline Electroencefalograma & $96(93,2 \%)$ \\
\hline Test de apnea & $71(68,9 \%)$ \\
\hline Potenciales evocados multimodales & $18(17,4 \%)$ \\
\hline Ecodoppler transcraneal & $17(16,5 \%)$ \\
\hline Evaluación del flujo cerebral por angiografía por TAC o RNM & $10(9,7 \%)$ \\
\hline \multicolumn{2}{|c|}{ Alteraciones que sobrevienen a la muerte encefálica $(\mathrm{N}=103)$} \\
\hline Disfunción hemodinámica & $65(63,2 \%)$ \\
\hline Diabetes insípida & $48(46,6 \%)$ \\
\hline Hipotermia & $47(45,6 \%)$ \\
\hline Hiperglucemia & $31(30,1 \%)$ \\
\hline Trastornos de coagulación & $19(18,4 \%)$ \\
\hline
\end{tabular}

TAC: tomografía axial computada; RNM: resonancia nuclear magnética. 


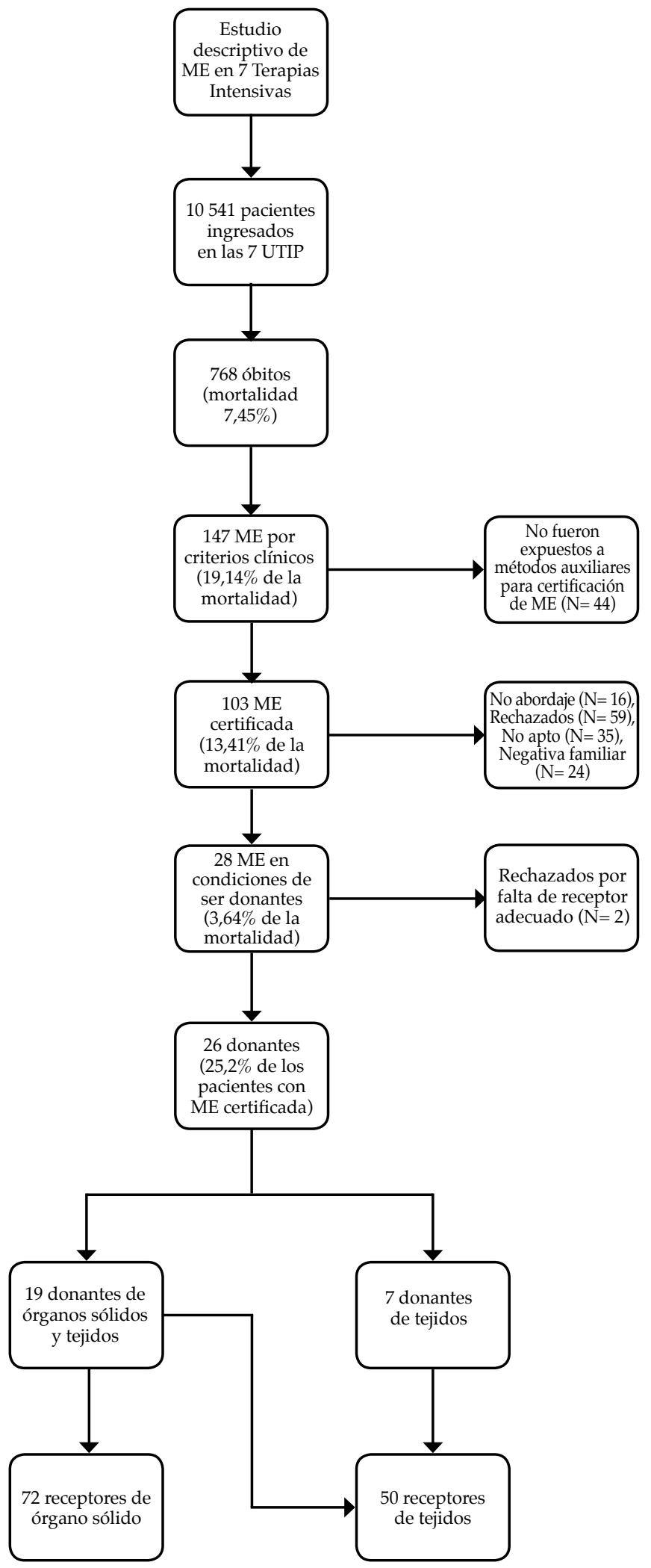

ME: muerte encefálica; UTIP: unidades de terapia intensiva pediátricas. 
presentaron 3 o más complicaciones asociadas.

De los 103 pacientes con ME certificada por MA, 16 fueron catalogados como NO ABORDADOS. De los 87 pacientes que sí fueron ABORDADOs por el INCUCAI e iniciaron el proceso como potenciales donantes, $59(67,8 \%)$ fueron descartados como donantes por el organismo supervisor, 35 casos por ser NO APTOS y 24 por NEGATIVA FAMILIAR. De los 28 casos de ME certificada que cumplían criterios para ser donantes, en 26, se llevó a cabo el procedimiento (mediana de 10 años de edad), mientras que los dos donantes restantes fueron rechazados por falta de receptor adecuado. De los 26 donantes, 19 fueron reales, definidos como aquellos quienes donaron órganos sólidos y tejidos, mientras que los restantes 7 pacientes fueron donantes solo de tejidos.

Se beneficiaron con el trasplante 72 pacientes que recibieron órganos sólidos, mientras que otros 50 pacientes fueron o serán potencialmente injertados con tejidos.

\section{DISCUSIÓN}

La incidencia de ME certificadas $(\mathrm{N}=103)$ en las 7 UTIP donde se desarrolló el trabajo fue del $1 \%$ de los ingresos, que representó el 13,4\% de la mortalidad, valores similares a los reflejados en otros trabajos internacionales ${ }^{3,9} \mathrm{y}$ en un estudio multicéntrico que se desarrolló en el país y abarcó 16 UTIP y determinó que la ME era responsable del 11\% de los óbitos (52 de 448). ${ }^{11}$

El politraumatismo fue la principal causa de ME, característica que comparten las publicaciones de González ${ }^{4}$ y Joffe, ${ }^{9}$ con mediana de edad de 7 años, muy por encima de la reflejada por Burns ${ }^{3}$ (2 años) y similar a las reportadas por Gündüz ${ }^{5}$ y el mencionado trabajo de Joffe. ${ }^{9}$

La certificación de la ME mediante MA es obligatoria en Argentina, ${ }^{1}$ en contraposición con EE. UU., Canadá, Australia y algunos países de Europa e Iberoamérica, ${ }^{12}$ en donde puede ser certificada por examen clínico. ${ }^{6}$ Cabe mencionar que, en el presente trabajo, el 30\% de los pacientes que presentaron clínica de ME (44 de 147) no fueron sometidos a certificación por los mencionados métodos, lo que alberga la posibilidad de potenciales donantes perdidos, cifra algo superior a la reportada por un trabajo multicéntrico desarrollado en Brasil, ${ }^{13}$ que reveló que un $20 \%$ de las ME no eran certificadas. Se considera que las implicancias de adoptar una política sanitaria referente a la obligatoriedad de los MA están sujetas a condicionamientos éticos y culturales inherentes a cada país y ameritan una discusión de la sociedad científica en su conjunto. En el presente trabajo, el electroencefalograma y el test de apnea fueron los métodos más utilizados para certificar la ME, eventualidad compartida en otras publicaciones. ${ }^{4,5,9}$

En lo referente a las horas que trascurren desde el diagnóstico de ME, en la casuística presentada, fue de $24 \mathrm{~h}$ (mediana). Estos valores son dispares en la bibliografía, ya que se observan $8 \mathrm{~h}$ en la serie de González ${ }^{4}$ y 6 días en la de Gündüz. ${ }^{5} \mathrm{La}$ importancia de la evaluación de esta variable se sustenta en las alteraciones que sobrevienen a la $\mathrm{ME}$, que requieren de intervenciones a fin de sostener la viabilidad de los órganos que serán objetos de ablación. En el presente trabajo, el $89 \%$ de las ME presentaron alteraciones; las más frecuentes fueron la disfunción hemodinámica, ${ }^{14}$ diabetes insípida e hipotermia, en coincidencia con la literatura. ${ }^{4,5}$ Otra arista que desnuda la determinación de las horas que transcurren desde el diagnóstico de ME es la dificultad de retirar el soporte vital en aquellos no donantes, particularidad reflejada en el trabajo de Althabe, ${ }^{11}$ en el que, en el $100 \%$ de los casos, se mantuvo el soporte ventilatorio y, en el $82 \%$, el sostén inotrópico.

$\mathrm{Al}$ inicio del período de estudio, el porcentaje de pacientes < de 18 años representaba el 1\% del total de la lista de espera del INCUCAI, que ascendió al 2,6\% al cierre del estudio, valores que comparten otros países, como EE. UU. (1,5\%) y el Reino Unido (2\%). ${ }^{6}$ Cabe destacar que el mencionado aumento no va aparejado al número de donantes, por lo que las necesidades insatisfechas se incrementan en este grupo etario.

En nuestra serie, el 25\% de los pacientes con ME certificada fueron donantes $(26 / 103)$ y el $18 \%$ lo hicieron con órganos sólidos (19/103), valor muy superior al trabajo de Lago, ${ }^{13}$ que reveló un total de 525 óbitos en 7 UTIP, $61 \mathrm{ME}$ y solo 6 donantes; mientras que, en el Reino Unido, ${ }^{15}$ según un estudio que abarcó 26 hospitales, con un total de $189 \mathrm{ME}(16,7 \%$ de los óbitos), se efectivizó la donación de órganos sólidos en el $24,3 \%$ de ellas. En dicho trabajo, ${ }^{15}$ los pacientes fueron clasificados en 2 grupos, aquellos con $\mathrm{ME}$, que incluyó a 37 donantes, y un segundo grupo, catalogado como "corazón no latiente", que contribuyó con 9 donantes; cifras superiores se evidencian en el reporte de Morris ${ }^{16}$ de 25 UTIP del Reino Unido en pacientes con traumatismo craneoencefálico grave, en el que 12 de 26 pacientes con ME fueron donantes. 
En nuestra serie, se lograron implantar 72 órganos sólidos, provenientes de 19 donantes, con una relación receptor/donante cercana a 4 , comparable a la serie de Ream, ${ }^{8}$ quien dividió el rendimiento de órganos según el grupo etario: de 3,5 y 4,9 para los grupos 0-10 y 11-17 años, respectivamente. Esta relación en la serie de Brierley ${ }^{15}$ fue de 2,5 (114 receptores / 46 donantes).

La negativa familiar fue motivo de rechazo en el $40 \%$ de los casos (24/59), que fue superior a otras series (20-30\%). ${ }^{9,10,17}$ Las causas de dicha negativa no están analizadas en el presente trabajo, pero impresionaría que refleja una falta de concientización y educación en la sociedad, y obliga a unificar esfuerzos a fin de revertir esta tendencia, con la necesidad de acceder a la opinión por parte de los familiares sobre la donación en forma previa.

La procuración de órganos es el proceso de obtención de órganos y tejidos para trasplante con el objetivo de dar respuesta a las personas en lista de espera. Está compuesto por varios pasos logísticos de diversa índole, con un ordenamiento sistematizado, que requieren de acciones coordinadas en cada una de sus etapas. Las distintas líneas de investigación referentes al proceso de gestión de órganos persiguen optimizarlo a fin de paliar la demanda insatisfecha. En este sentido, en Argentina, se han puesto en marcha diferentes programas para identificar posibles donantes, uno de ellos fue el Coordinador Hospitalario, con el cual se duplicó el número de procuraciones en el período 2003-2010, pero luego presentó una tendencia decreciente. En 2013, se puso en marcha el programa Hospital Donante, como una estrategia para institucionalizar la actividad de procuración en los hospitales; sin embargo, los resultados no lograron el cambio cultural en el sistema de salud. Así, el nuevo plan del ente regulador es lograr que el modelo extrahospitalario de procuración mute a una modalidad en la que el hospital asuma integralmente el proceso donación-trasplante. Esto ya está implementado en otros países, ${ }^{8}$ donde son múltiples las organizaciones que gestionan la procuración de órganos.

Cabe mencionar que, al momento del cierre del período de estudio, la lista de espera para ser trasplantados ascendía a 288 pacientes menores de 18 años; 57 requerían córneas o escleróticas y 231 órganos sólidos, y el 32\% eran menores de 10 años.

El presente trabajo pretende realizar un diagnóstico de situación en relación con el proceso de gestión de órganos en la Argentina, con la particularidad de no existir en la bibliografía nacional otro trabajo que repare en la eficacia de dicho proceso y la limitante de acceder a dichos resultados en forma retrospectiva, con la posibilidad de albergar subregistro.

\section{CONCLUSIÓN}

La ME clínica representó el 19,1\% de los óbitos, mientras que la ME certificada, el 13,4\%. Un 15,5\% de las ME certificadas no fueron abordadas por el ente regulador.

Los donantes efectivos, aquellos en los cuales se completó el proceso de trasplante, representaron el 25\% de los pacientes con ME certificada (26/103); en 19 de los 26 casos, se efectivizó el trasplante de órganos sólidos y tejidos y, en 7 casos, solo de tejidos.

\section{REFERENCIAS}

1. Abaroa L, Garretto N. Muerte encefálica. Situación legal en Argentina. Neurolog Argent 2013;5(2):101-7.

2. Protocolo nacional para certificar el diagnóstico de muerte bajo criterios neurológicos (muerte encefálica). Ministerio deSalud. Resolución N ${ }^{\circ} 275$ / 2010 - Boletín oficial 2010;31844. [Consulta: 18 de agosto de 2016]. Disponible en: http: / / www.cucaiba.gba.gov.ar/viejo/2013/pdf_2013/2013_ protocolo_muerte.pdf.

3. Burns J, Sellers D, Meyer E, et al. Epidemiology of Death in the PICU at Five U.S. Teaching Hospitals. Crit Care Med 2014;42(9):2101-8.

4. González N, Fernández M, Galán C, et al. Muerte encefálica y donación en población infantil. An Pediatr (Barc) 2004;60(5):450-3.

5. Gündüz R, Şahin S, Uysal-Yazıcı M, et al. Brain death and organ donation of children. TurkJ Pediatr 2014;56(6):597-603.

6. Brierley J, Hasan A. Aspects of deceased organ donation in paediatrics. Br J Anaesth 2012;108 (Suppl 1):i92-5.

7. Workman J, Craig WM, Meyers R, et al. Pediatric Organ Donation and Transplantation. Pediatrics 2013;131(6): e1723-30.

8. Ream R, Armbrecht E. Survey of U.S. Organ Procurement Organizations Regarding Pediatric Organ Donor Management. Pediatr Crit Care Med 2016;17(10):e459-68.

9. Joffe A, Shemie S, Farrell C, et al. Brain death in Canadian PICUs: Demographics, timing, and irreversibility. Pediatr Crit Care Med 2013;14(1):1-9.

10. Toida C, Muguruma T. Pediatric brain death in a Japanese pediatric hospital. Acute Med Surg 2016;3(1):10-5.

11. Althabe M,Cardigni G, VassaloJ, etal. Dying in the Intensive Care Unit: collaborative multicenter study about forgoing life-sustaining treatment in Argentine Pediatric Intensive Care Units. Pediatr Crit Care Med 2003;4(2):164-9.

12. Escudero D, Matesanz R, Soratti C, et al. Muerte encefálica en Iberoamérica. Med Intensiva 2009;33(9):415-23.

13. Lago P, Piva J, Garcia P, et al. Brain death: medical management in seven Brazilian pediatric intensive care units. J Pediatr (Rio J) 2007;83(2):133-40.

14. Krishnamoorthy V, Borbely X, Rowhani-Rahbar A, et al. Cardiac dysfunction following brain death in children: prevalence, normalization, and transplantation. Pediatr Crit Care Med 2015;16(4):e107-12.

15. Brierley J. Paediatric organ donation in the UK. Arch Dis Child 2010;95(2):83-8.

16. Morris K, Tasker R, Parslow R, et al. Organ donation in pediatric traumatic brain injury. Intensive Care Med 2006;32(9):1458.

17. Tsai E, Shemie S, Cox P, et al. Organ donation in children: role of the pediatric intensive care unit. Pediatr Crit Care Med 2000;1(2):156-60. 